Title : The 5th Zermatt ISM Symposium

Editors : Conditions and impact of star formation

EAS Publications Series, 2019

\title{
SPICA: THE NEXT GENERATION INFRARED SPACE TELESCOPE
}

\author{
Javier R. Goicoechea ${ }^{1}$ and Takao Nakagawa ${ }^{2}$ on behalf of the \\ SAFARI/SPICA teams
}

\begin{abstract}
We present an overview of SPICA, the Space Infrared Telescope for Cosmology and Astrophysics, a world-class space observatory optimized for mid- and far-IR astronomy (from 5 to $\sim 210 \mu \mathrm{m}$ ) with a cryogenically cooled $\sim 3.2 \mathrm{~m}$ telescope $(<6 \mathrm{~K})$. Its high spatial resolution and unprecedented sensitivity in both photometry and spectroscopy modes will enable us to address a number of key problems in astronomy. SPICA's large, cold aperture will provide a two order of magnitude sensitivity advantage over current far-IR facilities ( $\lambda>30 \mu \mathrm{m}$ wavelength). In the present design, SPICA will carry midIR camera, spectrometers and coronagraph (by JAXA institutes) and a far-IR imager FTS-spectrometer, SAFARI $(\sim 34-210 \mu \mathrm{m}$, provided by an European/Canadian consortium lead by SRON). Complementary instruments such as a far-IR/submm spectrometer (proposed by NASA) are also being discussed. SPICA will be the only space observatory of its era to bridge the far-IR wavelength gap between JWST and ALMA, and carry out unique science not achievable at visible or submm wavelengths. In this contribution we summarize some of the scientific advances that will be made possible by the large increase in sensitivity compared to previous infrared space missions.
\end{abstract}

\section{Introduction}

Understanding of the origin and evolution of galaxies, stars, planets, our Earth and of life itself are fundamental objectives of Science in general and Astronomy in particular. Although impressive advances have been made in the last twenty years, our knowledge of how the Universe has come to look as it does today is far

\footnotetext{
${ }^{1}$ Departamento de Astrofísica. Centro de Astrobiología (CSIC-INTA), Madrid, Spain

${ }^{2}$ Institute of Space and Astronautical Science (ISAS) and Japan Aerospace Exploration Agency (JAXA). Yoshinodai, Sagamihara, Kanagawa 229-8510, Japan.
}

(C) EDP Sciences 2019

DOI: (will be inserted later) 
Title : The 5th Zermatt ISM Symposium

from complete. A full insight of the processes involved is only possible with observations in the long wavelength infrared waveband of the electromagnetic spectrum. It is in this range that astronomical objects emit most of their radiation as they form and evolve in regions where obscuration by dust prevents observations in the visible and near infrared. Over the past quarter of a century successive space infrared observatories (IRAS, ISO, Spitzer and AKARI) have revolutionized our understanding of the evolution of stars and galaxies. Mid to far infrared observations have led to stunning discoveries such as the Ultra Luminous Infrared Galaxies (ULIRGS), the basic processes of star formation from "class 0" protostars through to the clearing of the gaseous proto-planetary disks and the presence of dust excesses around main sequence stars. The Herschel Space Observatory launched in 2009 continues this work in the far infrared and sub-mm and JWST, due for launch around 2014, will provide a major boost in observing capability in the $2-28 \mu \mathrm{m}$ range. Previous infrared missions have been hampered by the requirement to cool the telescope and instruments to $<5 \mathrm{~K}$ using liquid cryogens. This has limited the size of the apertures to $<1 \mathrm{~m}$ and our view of the infrared Universe has been one of poor spatial resolution and limited sensitivity. The Herschel mission addresses the first of these by employing a $3.5 \mathrm{~m}$ mirror to dramatically increase the available spatial resolution but, because it is only cooled to $\sim 80 \mathrm{~K}$ (Pilbratt et al. 2010), only offers a modest increase in sensitivity in the 55-210 $\mu \mathrm{m}$ range compared to previous facilities. JWST will provide a major increase in both spatial resolution and sensitivity but only up to $28 \mu \mathrm{m}$.

\section{SPICA}

The JAXA led mission SPICA is an observatory that will provide imaging and spectroscopic capabilities in the 5 to $210 \mu \mathrm{m}$ wavelength range with a $\sim 3.2 \mathrm{~m}$ telescope (current design), but now cooled to a temperature of $\sim 6 \mathrm{~K}$. In combination with a new generation of highly sensitive detectors, the low telescope temperature will allow us to achieve sky-limited sensitivity over the full 5 to $210 \mu \mathrm{m}$ band for the first time. This unique capability means that SPICA will be between one and two orders of magnitude more sensitive than Herschel in the far infrared band. SPICA will cover the full 5 to $210 \mu \mathrm{m}$ wavelength range, including the missing $28-55 \mu \mathrm{m}$ octave which is out of the Herschel and JWST domains, with unprecedented sensitivity and spatial resolution. Furthermore, SPICA will be the only observatory of its era to bridge the wavelength gap between JWST and ALMA, and carry out unique science not achievable at (sub)mm wavelengths with ALMA. In the mid infrared SPICA will be able to carry out medium and high-resolution $(R \sim 30,000)$ spectroscopy, one order of magnitude higher than in JWST, and will include spatial high multiplexing imaging and medium spectral resolution capabilities. In addition, the characteristics of the SPICA monolithic telescope will provide unique and optimal conditions for mid infrared coronagraphy in imaging and, uniquely, spectroscopic mode. 


\subsection{Scientific Instruments}

At present, the scientific instruments foreseen for SPICA are:

- A mid infrared camera and spectrophotometer, MIRACLE (5-38 $\left.\mu \mathrm{m}, 6^{\prime} \times 6^{\prime} \mathrm{FOV}\right)$.

- A mid infrared medium resolution spectrometer, MIRMES $(10.32-36.04 \mu \mathrm{m}$ with a spectral resolution of $R \sim 900-1500)$.

- A mid infrared high resolution spectrometer, MIRHES (4-18 $\mu \mathrm{m}$ with a spectral resolution of $R \sim 20,000-30,000)$.

- A mid infrared coronagraph, SCI (5(3.5)-27 $\mu \mathrm{m}, R \sim 20-200$, contrast $10^{-6}$, inner working angle $\sim 3.3 \lambda / \mathrm{D}_{t e l}$ ).

- SAFARI, a far infrared imaging Fourier-Transform Spectrometer designed to provide continuous coverage in photometry and spectroscopy from 34 to $210 \mu \mathrm{m}$, with a field of view of $2^{\prime} \times 2^{\prime}$ and spectral resolution modes of $R \sim 2000$ (at $100 \mu \mathrm{m}$ ), $R$ a few hundred and $20<R<50$. The spectral sensitivity is required to be $\sim 3.5 \times 10^{-19} \mathrm{~W} \mathrm{~m}^{-2}$ at $48 \mu \mathrm{m}(5 \sigma, 1$ hour $)$. TES bolometer detectors will be used. SAFARI is to be built by a consortium of European institutes lead by SRON (with Canadian and Japanese participation).
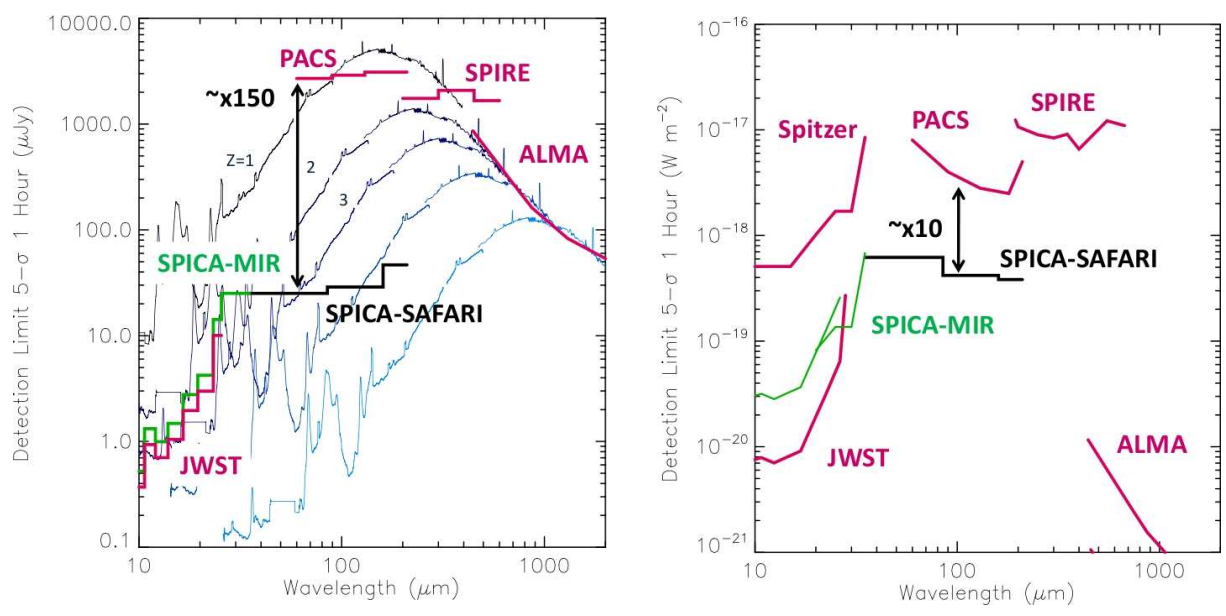

Fig. 1. Left panel: Photometric performance expected for SPICA (green and black), compared to Herschel, ALMA and JWST (purple), for a point source (in $\mu \mathrm{Jy}$ for $5 \sigma-1 \mathrm{~h}$ ). Note the $\sim 2$ orders of magnitude increase in far-IR photometric sensitivity compared to Herschel-PACS. For illustrative purposes the SED of the starburst galaxy M82 as redshifted to the values indicated is shown in the background. Right panel: Spectroscopic performance expected for SPICA (green and black) compared to predecessor and complementary facilities (purple) for an unresolved line for a point source (in $\mathrm{Wm}^{-2}$ for $5 \sigma-1 \mathrm{~h}$ ). For ALMA, $100 \mathrm{~km} \mathrm{~s}^{-1}$ resolution is assumed. The SPICA MIR sensitivities are scaled by telescope area from the JWST and Spitzer-IRS values respectively. SPICA/SAFARI sensitivity figures adapted from Swinyard, Nakagawa et al. (2009). 
Title : The 5th Zermatt ISM Symposium

\subsection{Science with SPICA}

SPICA offers a sensitivity up to two orders of magnitude better than Herschel covering the mid-to-far-IR (mostly unreachable from the ground) with imaging, spectroscopic and coronagraphic instruments (see Figure 1). Thanks to this tremendous increase in sensitivity, SPICA will make photometric images in a few seconds that would take hours for Herschel and will take a full 5-210 $\mu \mathrm{m}$ infrared spectrum of an object in one hour that would take several thousand hours for Herschel. We illustrate this major increase in sensitivity in Figure 2 which shows the area covered by a full spatial and spectral survey in which we can detect spectroscopically all galaxies down to a luminosity of $\sim 10^{11} \mathrm{~L}_{\odot}$ at $\mathrm{z}=1$ and $\sim 10^{12} L_{\odot}$ at $\mathrm{z}=2$ in 900 hours. In theory, in about twice this time, the Herschel-PACS spectrometer would just be able to detect a single object over its full waveband to the same sensitivity. We can immediately see that this major increase in sensitivity, combined with a wide field of view and coverage of the full $5-210 \mu \mathrm{m}$ waveband, will revolutionize our ability to spectroscopically explore the nature of the thousands of objects that Herschel, JWST, and SPICA will discover in photometric surveys.

With its powerful scientific capabilities, SPICA will provide unique and groundbreaking answers to many key questions, and it is with this goal that the SPICA science objectives are being defined:

(i) The formation and evolution of planetary systems: By accessing key spectral diagnostic lines, SPICA will provide a robust and multidisciplinary approach to determine the conditions for planetary systems formation. This will include the detection for the first time of the most relevant species and mineral components in the gas and dust of hundreds of transitional protoplanetary disks at the time when planets form. SPICA will also be able to trace the warm gas in the inner $(<30 \mathrm{AU})$ disk regions and by resolving the gas Keplerian rotation (e.g., by resolving the mid-IR $\mathrm{H}_{2}$ lines), will allow us to observe the evolution of disk structure due to planet formation. SPICA will study debris disks and make the first unbiased survey of the presence of zodiacal clouds in thousands of exoplanetary systems around all stellar types. It will allow us to detect both the dust continuum emission and the brightest grain/ice bands as well as the brightest lines from any gas residual present in the disk. SPICA will have the unique capability to observe water ice in all environments, and thus fully explore its impact on planetary formation and evolution and in the emergence of habitable planets. In the closest debris disks, SPICA will spatially resolve the distribution of water ice and determine the position of the "snow line" around Vega-like stars, which separates the inner disk region of terrestrial planet formation from that of the outer planets. SPICA will also drastically enhance our knowledge of the Solar System by making the first detailed characterization of hundreds of Kuiper Belt Objects, and of different families of inner, hotter centaurs, comets and asteroids. SPICA will provide the means to quantify their composition and determine unambiguously their size distribution: critical observational evidence for the models of Solar System formation. No other planned or present facility will be able to carry 
out these observations. SPICA will provide direct imaging and low resolution mid infrared spectroscopy of outer young giant exoplanets (e.g., at $\sim 10 \mathrm{AU}$ of a star at $\sim 10 \mathrm{pc}$ ), which will allow us for the first time to study the physics and composition of their atmospheres in a wavelength range particularly rich in spectral signatures (e.g., $\mathrm{H}_{2} \mathrm{O}, \mathrm{CH}_{4}, \mathrm{O}_{3}$, silicate clouds, $\mathrm{NH}_{3}, \mathrm{CO}_{2}$ ) and to compare it with Solar System planet atmospheres. In addition, mid infrared transit photometry and high resolution spectroscopy of "hot Jupiters" will be routine with SPICA. Finally, by mapping very extended but faint regions, SPICA will also provide an unprecedentedly sensitive window into key aspects of the dust life-cycle both in the Milky Way and in nearby galaxies, from its formation in evolved stars, its evolution in the ISM, its processing in supernova-generated shock waves and massive stars, to its final incorporation into star forming cores and protoplanetary disks.

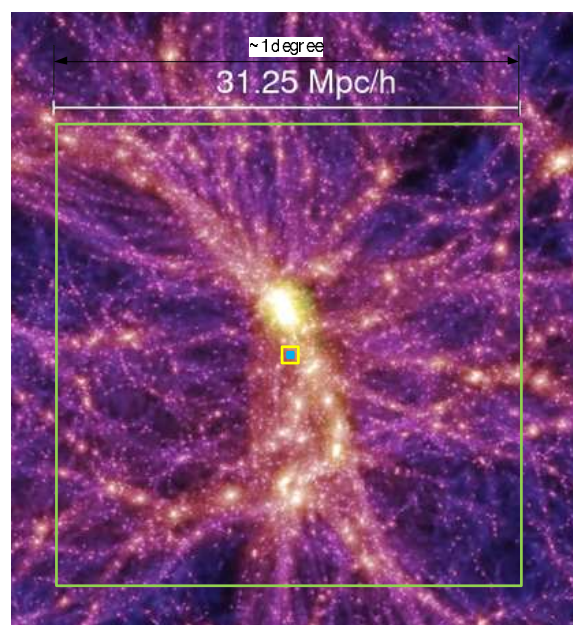

Fig. 2. Spectroscopic mapping speeds of the SPICA far-IR instrument (SAFARI) and Herschel- PACS superposed on a realization of the Millenium simulation at $\mathrm{z} \sim 1.4$ (Springel et al. 2006). In the center are the footprints of the instantaneous spectroscopic FOV of PACS (blue) and SAFARI (yellow). The large green box shows the area covered by SAFARI in a 900 hour spectral full wavelength spectral survey ( $\sim 1$ degree).

(ii) The formation and evolution of galaxies: SPICA observations will provide a unique insight into the basic questions about how galaxies form and evolve such as: What drives the evolution of the massive, dusty distant galaxy population, and what feedback/interplay exists between the physical processes of mass accretion and star formation? How and when do the normal, quiescent galaxies such as our own form, and how do they relate to (Ultra) Luminous Infrared Galaxies (ULIRGs)? How do galaxy evolution, star formation rate and AGN activity vary with environment and cosmological epoch? Substantial progress in this area can only be made by making the transition from large-area photometric to large-area spectroscopic surveys in the mid to far infrared, which will be possi- 
ble with SPICA. This is because the mid and far infrared region plays host to a unique suite of diagnostic lines to trace the accretion and star formation, and to probe the physical and chemical conditions in different regimes from AGN to star-forming regions. While the Herschel-PACS spectrometer detects the brightest far infrared objects at $\mathrm{z} \sim 1$, SPICA will be able to carry out blind spectroscopic surveys out to $\mathrm{z} \sim 3$. This will lead to the first statistically unbiased determination of the co-evolution of star formation and mass accretion with cosmic time. Spectroscopic surveys will provide direct and unbiased information on the evolution of the large scale structure in the Universe from $\mathrm{z} \sim 3$ and the unprecedented possibility to investigate the impact of environment on galaxy formation and evolution as a function of redshift. The high sensitivity of SPICA will enable photometric surveys beyond $\mathrm{z} \sim 4$ that will resolve more than $90 \%$ of the Cosmic Infrared Background (in comparison with $50 \%$ that Herschel will achieve). SPICA will also observe Milky Way type galaxies in the far infrared out to $\mathrm{z} \sim 1$, where the cosmic star formation rate peaks. For the first time, we will be able to piece together the story of the evolution of our own galaxy and answer the question of whether we are in a "special place" in the cosmos.

By observing at far-IR wavelengths, Herschel is presently providing spectacular results in many fields of Astronomy. A new mission reaching better sensitivity in the mid- and far-IR domain must therefore follow to continue this work and gain a deeper understanding of the physics of the objects discovered by Herschel: i.e., to spectroscopically measure star formation rates in the obscured extragalactic sources at different cosmic epochs and explore the physics and chemistry of planet formation. To obtain this increase in sensitivity we need a cold $(<6 \mathrm{~K})$ telescope. SPICA promises this in 2018-2020.

SPICA will be an international mission and will be open to the worldwide astronomical community. Japan is in charge of the whole integration of the system. The assessment study on the European contribution to the SPICA project has been carried out under the framework of the ESA Cosmic Vision (SPICA Assessment Study Report for ESA Cosmic Vision 2015-2025 Plan, see 2010arXiv1001.0709S for details). US and Korean participation are also being discussed.

Acknowledgments: We warmly thank Kate Isaak, Bruce Swinyard, Ana Heras and the SPICA/SAFARI science teams for fruitful discussions over the last years. J.R.G. was supported by a Ramón y Cajal contract and by the AYA2009-07304 and CSD2009-00038 grants from the Spanish MICINN.

\section{References}

Pilbratt, G. L. et al. 2010, $A \mathscr{E} A$, 518, L1.

Springel, V., Frenk, C.S. \& White, S.D.M. 2006, Nature, 440, 1137.

SPICA Study Team Collaboration, 2010. SPICA Assessment Study Report for ESA Cosmic Vision 2015-2025 Plan, arXiv1001.0709S.

Swinyard, B., Nakagawa, T., et al. 2009, Experimental Astronomy, 23, 1, 193-219. 